\title{
Nordiques
}

39 | 2020

Varia

\section{Modernité de la série Mästerdetektiven Kalle Blomkvist d'Astrid Lindgren}

Harri Veivo

\section{OpenEdition}

Journals

Édition électronique

URL : http://journals.openedition.org/nordiques/570

DOI : $10.4000 /$ nordiques. 570

ISSN : 2777-8479

Éditeur :

Association Norden, Bibliothèque de Caen la mer

Référence électronique

Harri Veivo, « Modernité de la série Mästerdetektiven Kalle Blomkvist d'Astrid Lindgren », Nordiques [En ligne], 39 | 2020, mis en ligne le 01 novembre 2020, consulté le 13 mars 2021. URL : http:// journals.openedition.org/nordiques/570 ; DOI : https://doi.org/10.4000/nordiques.570

Ce document a été généré automatiquement le 13 mars 2021.

Nordiques 


\title{
Modernité de la série
}

\section{Mästerdetektiven Kalle Blomkvist d'Astrid Lindgren}

\author{
Harri Veivo
}

1 Le succès mondial de la littérature d'enfance et de jeunesse nordique tire son origine de la réflexion multiple - philosophique, politique, littéraire et artistique - sur les conceptions de la famille et du «chez soi » menée par des intellectuels et créateurs comme Ellen Key et Carl Larsson ${ }^{1}$ vers le tournant du $\mathrm{XX}^{\mathrm{e}}$ siècle ainsi que des romans, récits et bandes dessinées novateurs publiés à partir de 1945 par Tove Jansson, Lennart Hellsing et Astrid Lindgren, trois auteurs qui, s'appuyant sur des précurseurs comme Selma Lagerlöf et Zacharias Topelius, ont modernisé le genre dans la littérature suédophone. De cette dernière, Astrid Lindgren, on a retenu avant tout la jeune hérö̈ne anarchique Fifi Brindacier, véritable icône suédoise, mais aussi Ronya la fille de brigand, les frères Cœur-de-lion, Rasmus, Vic le Victorieux et Zozo (connus en France aussi sous leurs noms d'origine Karlsson et Emil). Ces figures légendaires ont fait de Lindgren l'auteur suédois le plus traduit aux $\mathrm{XX}^{\mathrm{e}}$ et $\mathrm{XXI}^{\mathrm{e}}$ siècles ${ }^{2}$.

2 Les aventures du maître détective Kalle Blomkvist semblent avoir moins bien résisté au temps. La série en trois volumes, Mästerdetektiven Blomkvist (1946, le seul à être traduit en français en 1948 avec le titre L'as des détectives), Mästerdetektiven lever farligt (Le Maître détective vit dangereusement, 1951) et Kalle Blomkvist och Rasmus (Kalle Blomkvist et Rasmus, 1953), a certes connu un succès mondial avec des traductions en une trentaine de langues, dont toutes les langues nordiques, l'allemand, le français, et l'anglais, mais aussi l'hébreu, le japonais, le chinois ou le géorgien ${ }^{3}$. Elle a été particulièrement bien reçue dans certains pays de l'ancien bloc de l'est, dont la République démocratique d'Allemagne et la République socialiste soviétique de l'Estonie, où le champ littéraire était très réceptif à son discours réaliste et sa mise en valeur d'un groupe d'enfants solidaire et héroïque, tous deux des éléments qui convenaient mieux que Fifi à l'esthétique officielle du réalisme socialiste ${ }^{4}$. Neuf adaptations au cinéma ou à la télévision ont vu le jour entre 1947 et 1997. Mais force est 
de constater que le succès de la série pâlit aujourd'hui en comparaison de l'intérêt que les lecteurs et les critiques portent aux autres romans de Lindgren et surtout à Fifi Brindacier. Si, dans le domaine international, Helena Forsås-Scott l'ignore, Maria Nikolajeva, Thierry Maricourt et Boel Westin ne la nomment qu'en passant et les auteurs de Beyond Pippi Longstocking s'intéressent en fait surtout à Fifi', la situation n'est guère meilleure en Suède où l'ouvrage collectif sur la littérature pour enfants Läs mig! Sluka mig! (Lis-moi! Dévore-moi!) et l'entrée sur Lindgren dans Kvinnornans litteraturhistoria (L'Histoire littéraire des femmes) par exemple la passent sous silence et Lena Kåreland ne la mentionne que très brièvement dans son manuel universitaire Skönlitteratur för barn och unga (Littérature pour enfants et jeunes). ${ }^{6}$

3 L'intérêt pour Fifi est bien entendu amplement mérité. Les livres que Lindgren a consacrés à son héroïne ont réussi à renouveler la littérature pour enfants de plusieurs manières. Comme l'a montré Kåreland dans son étude Modernismen i barnkammaren (Le modernisme dans la chambre des enfants), ils ont introduit de nouveaux éléments langagiers dans le discours littéraire, dont par exemple l'utilisation de l'argot et des jeux de mots qui renvoient aux apories de la philosophie du langage; ils ont introduit une figure de jeune fille qui affirme sa personnalité et sa volonté de vivre par des démarches artistiques, acrobatiques et comiques qui s'apparentent à l'esthétique du mouvement dada ; ils ont puisé dans la parodie et l'ironie pour remettre en question les structures de pouvoir dans la société. Dans ce sens, la sérié Fifi s'est positionnée résolument du côté du modernisme dans une situation de tension sur le champ littéraire où la vieille garde défendait la tradition et ses valeurs d'harmonie et de décorum et accusait les novateurs d'absence de talent et de goût et même de maladie psychique et de décadence. En même temps, elle a contribué à forger une nouvelle conception de l'enfant et de la fille qui continue à inspirer la pédagogie encore aujourd'hui. ${ }^{7}$

4 La série sur Kalle Blomkvist est plus réconciliatrice en comparaison avec le flamboyant radicalisme de Fifi. Elle propose cependant une réflexion complexe sur l'identité de l'enfant - ou de la jeune personne - et elle le fait via une modernité narrative et des thèmes qui, tout en restant moins explosifs que ce que le lecteur rencontre à Villa Drôle de repos, présentent un intérêt pour l'étude de la littérature suédoise et nordique, et notamment de l'autre genre à succès mondial venu du Nord, le polar. Un lien explicite mène en fait des trois volumes Blomkvist à la série Millennium de Stieg Larsson qui reprend deux figures de Lindgren, Kalle et Fifi, pour en proposer de nouvelles interprétations dans le couple asymétrique que forment Mikael Blomkvist et Lisbeth Salader : d'une part, le Mikael bosseur, sérieux et intégré à la société à l'image de Kalle, et de l'autre, la Lisbet solitaire, marginale et pourtant dotée de compétences exceptionnelles qui lui permettent de défier le pouvoir comme le faisait aussi Fifi. ${ }^{8} \mathrm{Au}$ delà de l'effet de " caisse de résonance culturelle $"^{9}$ de ces références aux personnages lindgreniens, on peut observer d'autres types de connexions entre les trois récits sur Blomkvist et le polar contemporain, notamment dans la conception de la Suède et des pays nordiques, envisagés comme un espace à part, moralement supérieur et protégé de l'influence extérieure - cristallisée sous la notion de "particularisme »- ainsi que dans l'hybridation de genres, la métatextualité et l'importance accordée à l'espace urbain et à la négociation de l'identité. L'intérêt de la série réside dans ces éléments que nous nous efforcerons d'analyser en montrant d'abord leur importance pour la 
poétique et la modernité de la série sur Kalle Blomkvist et en les reliant ensuite aux thèmes et problématiques caractéristiques au polar nordique contemporain.

\section{Affirmation et ambiguiité du particularisme suédois}

Astrid Lindgren a participé à un processus fondamental de renouvellement du champ littéraire suédois dans les années 1940 par l'intronisation de l'esthétique et la pensée moderne du XXe siècle dans la littérature pour enfants, et elle l'a fait en apportant un éthos fondamentalement positif et joyeux dans un contexte où le débat culturel et littéraire était dominé par des thèmes plus sombres de pessimisme et d'existentialisme qui reflétaient la catastrophe de la Seconde Guerre mondiale ${ }^{10}$. En même temps, elle a traité des thèmes d'actualité dans les discussions portant sur l'enfance, la jeunesse et la famille. Fifi est, dans les mots du chercheur suédois Ulf Boëthius, « wild, ill-mannered and disobedient", un mauvais élément qui a sa propre bande à elle; en plus, elle est orpheline et vit seule. Dans l'État-providence du folkhemmet (foyer du peuple), de tels éléments devaient être intégrés dans des institutions, dans un barnhem (orphelinat) ou - si le sujet était particulièrement difficile comme Fifi - dans un skyddshem (maison de correction). En offrant aux lecteurs une figure de jeune fille sauvage et mal élevée (par rapport aux attentes de l'époque), mais en même temps émancipée, solidaire et intelligente, Lindgren met en question ces conceptions normatives de la société suédoise sur la famille et l'enfance avec lesquelles elle-même, alors jeune mère d'un enfant né d'une relation extra-conjugale, avait dû se battre dans sa propre vie ${ }^{11}$.

La série Kalle Blomkvist offre une image plus réconciliante de l'enfance, de la famille et de la société. Les trois romans qui composent la série mettent en scène la vie de trois jeunes personnes de 13 ans, Kalle et ses amis Anders et Eva-Lotta, pendant des vacances d'été qui semblent ne jamais finir et qu'il faut remplir d'activités. Les trois héros traînent dans les rues et les jardins de la petite ville de Lillköping et jouent à la Guerre des Deux-Roses - inspirée de la guerre de succession entre les York et Tudor en Angleterre au $15^{\mathrm{e}}$ siècle (que Lindgren évoque aussi par une paraphrase du Henri IV de Shakespeare) - contre une bande de trois ennemis-amis dans la prairie et les ruines de château qui entourent la ville où, normalement, il ne se passe jamais rien. En même temps, Kalle rêve d'être un détective privé comme Hercule Poirot, doté de capacités de raisonnement extraordinaires et toujours alerte pour repérer tout individu ou événement suspect qui pourrait mettre en danger la société. Parfois, les jeunes doivent laisser leurs amis pour aider les parents dans leur travail ou dans leurs tâches ménagères. La préoccupation principale reste cependant le jeu; ils sont dans ce sens des enfants avant tout.

Dans chaque roman, les vacances d'été sont perturbées par un crime. Dans le premier livre de la série, trois cambrioleurs venus de Stockholm se disputent le partage du butin dans la petite ville de Kalle; dans le deuxième, un jeune homme endetté qui habite ailleurs tue son créancier à Lillköping; et dans le dernier, des espions au service d'une puissance étrangère kidnappent un professeur et son fils pour leur extorquer des formules secrètes qui sont la propriété de l'État suédois. Le mal vient ainsi toujours de l'extérieur. Les trois copains, mêlés à l'affaire par hasard, deviennent des témoins et des protagonistes dans sa résolution. À la fin, la police arrête les criminels et la Guerre des Deux-Roses reprend. 
$8 \mathrm{Vu}$ ainsi, la série Kalle Blomkvist appartient certainement à la grande tradition de la littérature comique dans la définition que le critique canadien Northrop Frye a donné à ce terme, c'est-à-dire qu'elle vise ultimement à l'intégration de la société ${ }^{12}$. Dans le monde de Kalle, le crime a son origine ailleurs. Il bouleverse la vie de la petite ville, mais tout retrouve sa place à la fin du récit. L'harmonie et la solidarité règnent et les conflits sont autrement absents comme dans une ville-exemple de l'État-providence suédois. La narration, qui respecte l'ordre chronologique de l'histoire et révèle au lecteur, par un jeu subtil de retardement typique au polar, toute l'information nécessaire pour se faire une représentation du monde fictif et comprendre le dénouement de l'intrigue, renforce cette fonction stabilisatrice. Cette vision réconciliatrice de la société reste cependant ambiguë. Elle repose sur une extériorisation du mal qui vise à démarquer un espace propre à part préservé du mal qui serait d'abord Lillköping et ensuite la Suède entière. Analysée de plus près, cette opération s'avère être sans fin, la frontière étant toujours un lieu qui sépare et unit en même temps.

Dans les deux premiers romans de la série, l'action est située à Lillköping uniquement et les criminels débarquent d'ailleurs pour déranger la vie paisible de la petite ville, alors que l'intrigue du troisième amène les enfants hors de la ville sur une île où les espions ont leur base secrète. L'origine du mal est ainsi déplacée graduellement de l'extérieur de la ville vers les pays étrangers. "Cela n'arrive tout simplement pas. Pas ici. Peut-être aux États-Unis - on pouvait en lire dans les journaux - mais non pas ici. ${ }^{13}$ Ainsi réagit Kalle dans le troisième volume de la série quand il devient le témoin de l'enlèvement d'un petit enfant. Émerge ainsi dans la série le constat de Lillköping d'abord et ensuite de la Suède entière comme un espace à part, préservé du mal et moralement supérieur au reste du monde. Cette conception du «particularisme suédois » est renforcée un peu plus loin dans le même livre où le crime est directement contrasté, à travers la perception et les pensées d'Eva-Lotta, avec le paysage suédois pour souligner son incompatibilité avec le contexte :

Elle capte tout avec son regard et le laisse ensuite vagabonder plus loin à travers la vitre. C'est bien à travers un paysage d'été suédois qu'ils foncent, aucun doute làdessus. Les champs de seigle mûr avec les marguerites et les bleuets sont décidément suédois. Les troncs de bouleaux blancs de même. Il n'y a que cette voiture et ses étranges passagers qui n'appartiennent pas ici, mais dans un film de gangsters américain. [...] Kidnappeurs - cela semble si bête dans un tel contexte suédois ensoleillé $!^{14}$

Pour Eva-Lotta, la criminalité est incompatible avec le paysage et la nature qui sont porteurs d'une identité nationale: soulignons en effet que les champs de seigle, les marguerites, les bleuets et les bouleaux non seulement appartiennent à un paysage suédois, mais ils sont qualifiés eux-mêmes de "suédois ». L'étonnement du jeune personnage annonce les réactions de Wallander et d'autres policiers de nos jours face aux crimes odieux, le «ce n'est pas possible ici » étant devenu un topos distinctif du polar nordique qui repose sur le "particularisme », c'est-à-dire sur la conception du territoire national comme un espace à part et moralement supérieur (ou en tout cas présupposé tel). L'anxiété que ce topos exprime pointe vers une réflexion sur la frontière qui sépare les "nous" des "eux » et forme un élément constitutif mais instable de la société; la frontière assumant la double fonction de fermeture et de communication en même temps. La série sur Kalle Blomkvist interroge la frontière entre le monde clos, connu et immuable de la petite ville et de la Suède rurale, décrite ici 
comme emblème de la nation, et l'espace extérieur où vivent des cambrioleurs professionnels, des personnes qui sont prêtes à tuer pour effacer les dettes qu'elles ont contractées, et des espions de pays hostiles - en bref, différents représentants du vaste monde opaque et conflictuel - dans le contexte de la guerre froide évoqué par l'intrigue $\mathrm{du}$ troisième volume. Le polar nordique contemporain continue le même questionnement dans le contexte actuel de globalisation des communications, des mobilités et des échanges commerciaux. Le traite des êtres humains, la prostitution, la contrebande, les drogues, le travail au noir sont des phénomènes qui traversent les frontières et qui sont impossibles à extérioriser, la demande sur le marché intérieur étant le moteur qui nourrit ces phénomènes ${ }^{15}$. Le polar moderne pointe ainsi vers une culpabilité collective : les crimes les plus odieux sont possibles «ici» aussi, et ils ne sont pas l'œuvre d'étrangers uniquement. La critique concerne ainsi potentiellement toute la société de l'État-providence. La série sur Blomkvist par contre restaure l'ordre dans la société et extériorise le mal, renforçant ainsi la conception de la Suède - et de Lillköping comme métonyme de la société suédoise idéalisée - comme un espace à part. Reste cependant le fait que, ici aussi, ce "particularisme » est relativisé par les liens que le crime tisse vers cet espace à part: dans le premier livre de la série, un des cambrioleurs est un cousin de la mère d'Eva-Lotta; dans le deuxième, le créancier habite à Lillköping; et dans le troisième, les espions, bien qu'au service d'un État étranger, sont Suédois. Dans la série sur Kalle Blomkvist comme dans le polar nordique, il est question de la relation entre l'intérieur et l'extérieur, entre le connu et l'inconnu, entre la communauté à laquelle on appartient et les autres. Les frontières sont poreuses, et les récits peuvent se lire comme une réflexion sur l'insécurité fondamentale que cette perméabilité crée.

\section{Hybridation de genres et métatextualité}

11 Dans la série Kalle Blomkvist, la modernité poétique typique à Lindgren - que Kåreland voit surtout dans Fifi - réside dans le dépassement des limites imposées par le roman à énigme classique où le déroulement de l'action se construisait à partir d'un mystère à résoudre. Ce sous-genre du polar a dominé la production suédoise et nordique jusqu'à la publication de Rosanna de Sjöwall et Wahlöö en 1965. Si le roman à énigme repose, selon Siefried Krakauer, sur «l'idée de la société civilisée parfaitement rationalisée » et représentée "sous une forme stylisée par la réfraction esthétique ${ }^{16}$, Maj Sjöwall et Per Wahlöö ont remis en question la rationalité même dans le fonctionnement de la société et dans le travail de la police tout en ouvrant le roman à d'autres zones de l'existence plus tristes et mornes que celles reprises par la stylisation esthétique de Conan Doyle, Agatha Christie et d'autres maîtres du roman à énigme. Si, en Suède dans les années 1950, Maria Lang, la reine du roman à énigme, pouvait commencer un roman par une liste de personnages représentant des types sociaux - le colonel à la retraite, la vieille fille qui fait des commérages, l'homme d'affaires fortuné et ainsi de suite ${ }^{17}$ - et si cette liste constituait la petite société parmi laquelle on allait trouver le coupable, cette option n'était plus possible pour Sjöwall et Wahlöö qui décrivaient un monde plus opaque et ouvert.

Dans la série sur Kalle Blomkvist, le monde reste certes familier en principe, mais le dépassement de la "réfraction esthétique " - il faut entendre par cette notion une opération de simplification à des fins esthétiques - s'opère par un mélange de genres 
qui engendre des intersections entre le jeu et le crime, l'imaginaire et la réalité, l'aventure et le cauchemar, et le monde des enfants et le monde des adultes. Les romans de la série utilisent des éléments narratifs et thématiques non seulement du roman à énigme, mais aussi du roman d'aventures, du roman historique et, dans le troisième volume, du roman d'espionnage. Si l'hybridation des genres est caractéristique à l'œuvre de Lindgren en général ${ }^{18}$, elle est également fondamentale dans les trois livres sur Kalle Blomkvist.

C'est en se laissant emporter par l'aventure et l'histoire ludique et imaginée pour aller explorer le manoir abandonné ou le château en ruines situé à la lisière de la ville que Kalle et ses amis sont confrontés au crime. À ces moments, la fantaisie rencontre la réalité, et le mode narratif dominant bascule du récit d'aventures au roman policier, sans que l'aventure ou l'histoire disparaissent pour autant. D'une part, elles fournissent au récit des schémas narratifs - comme l'exploration, la confrontation, la quête de Stormumriken (une sorte de Graal dans la Guerre des Deux-Roses) - qui font avancer la narration sur l'élucidation du crime, et, d'autre part, elles offrent aux personnages des valeurs et des outils cognitifs - par exemple, la solidarité, la camaraderie, le courage, et le code qu'ils utilisent pour communiquer entre eux - qui les aident à surmonter les épreuves dans le jeu et dans la réalité. En fait, le succès dans le récit policier repose sur la capacité des enfants à puiser dans les réserves offertes par le jeu et l'imagination. Ils vivent dans deux mondes en même temps, et l'hybridation des genres ouvre justement cet espace d'intersection à explorer dans les livres.

En même temps, la découverte du crime opère une autre modulation. Dans ses heures perdues, Kalle rêve d'être un maître détective du même rang que d'illustres prédécesseurs comme Sherlock Holmes et Hercule Poirot. Il s'ennuie sans sa petite ville paisible - «Pourquoi certains auraient-ils la chance de naître dans les bidonvilles de Londres ou les milieux criminels de Chicago où la mort et les coups de feu sont monnaie courante ? ${ }^{19}$, se demande-t-il, jaloux quand il s'ennuie dans sa vie paisible -, il nourrit son imagination de lectures et du cinéma, et consacre de grands efforts à l'observation des individus qu'il rencontre dans l'espoir de trouver quelque chose de louche. Quand le crime arrive, la triple relation entre les modèles littéraires, la vie imaginée par Kalle et la réalité devient problématisée de deux manières.

Premièrement, Kalle comprend que les autres - et surtout les criminels - peuvent puiser dans le même réservoir de récits pour déjouer les efforts de la police. La réalité et les récits véhiculés par la littérature et les médias sont ainsi imbriqués : notre conception du monde est façonnée par des textes et des systèmes de représentation collectifs. Le dernier volume va même jusqu'à suggérer que la réalité pourrait être textuelle :

« Oui », consentit Anders, « ce qui nous arrive ici, cela n'arrive que dans les livres. »

«Peut-être que ceci n'est qu'un livre », dit Kalle.

"Qu'est-ce que tu veux dire? Tu es un peu stupide?", répondit Anders.

"Peut-être que nous n'existons pas », continua Kalle d'un air rêveur. "Nous ne sommes peut-être que deux gars dans un livre que quelqu'un a imaginé. »

"Oui, toi éventuellement», dit Anders énervé. «Cela ne m'étonnerait pas que tu sois une faute de frappe, toute chose considérée. Mais moi non, je peux te l'assurer!»

«Tu ne le sais pas », soutint Kalle. «Peut-être que tu n'existes que dans un livre que j'ai imaginé. »

"Laisse tomber", dit Anders. "Si c'est le cas, tu existes dans un livre que j'ai 
imaginé, et je commence à regretter de t'avoir inventé. »

«D'ailleurs, j'ai faim », dit Kalle. ${ }^{20}$ par exemple. Le jeu métatextuel qui ouvre le mirage d'une textualité infinie est cependant interrompu par la dure réalité: la faim, c'est-à-dire les besoins physiologiques, le corps. La série propose dans son ensemble une réflexion sur la relation entre l'imaginaire - les récits que les romans policiers et le cinéma offrent - et la réalité où l'extension et la force d'attraction du premier est limité et contrecarré par les dures contraintes de la deuxième.

La deuxième problématisation s'opère quand Kalle exerce son discernement et ses capacités de raisonnement et d'action apprises dans les livres et réussit effectivement à aider la police, mais s'aperçoit en même temps de la distance qui sépare sa rêverie à la dure réalité de son identité : il est, finalement, juste Kalle Blomkvist, un garçon de 13 ans, et non pas un nouveau Poirot. Dans l'ensemble, le ton dans les romans reste léger et l'élucidation du crime est constamment interrompue par les joutes entre les Roses Blanches et Rouges ou par d'autres jeux, mais l'intersection entre polar et aventure permet cependant une sorte d'anagnorisis dans l'expérience de la limite entre le monde des enfants et celui des adultes. Kalle n'est certes pas un " homme tourmenté » comme ses illustres successeurs Martin Beck et Kurt Wallander qui souffrent de nombreuses incompatibilités qui traversent leur être (impossibilité de vivre une vie professionnelle et familiale en même temps, conflit entre les codes moraux intériorisés et les contraintes de la profession, etc.), mais on peut cependant observer que lui aussi fait l'expérience d'un décalage insurmontable entre une identité projetée - ce qu'il voudrait être - et la réalité de son être biologique et social. Parfois, il est même envahi par une sorte de sentiment de lassitude et de désarroi quand il n'est pas capable d'arrêter de se prendre pour un détective. Il doute de ses capacités, de son rôle, de sa légitimité. A la fin, il peut se replier dans le monde des enfants, celui du jeu, de l'imaginaire, de l'amitié, de l'innocence. Or, cette possibilité n'existe plus dans le polar contemporain.

\section{Le Lillköping des enfants : espace et identité}

18 Il est intéressant de noter que dans le passage que nous avons cité plus haut, Kalle exprime son regret de ne pas être né à Londres ou à Chicago. Il met ainsi en contraste les jungles urbaines étrangères, sources de fascination pour le jeune protagoniste, et la petite ville de Lillköping, ennuyeuse et prévisible. La série dans son ensemble offre cependant une image plus complexe de ce lieu idyllique. L'espace romanesque de Lillköping est constitué de zones qui sont emboîtées - comme la chambre de Kalle, située dans la maison de ses parents, à son tour entourée par un jardin, et ainsi de suite - ou bien juxtaposées - comme le jardin de Kalle qui permet d'accéder au jardin d'EvaLotta qui communique à son tour avec la rivière. L'ensemble constitue ainsi un réseau d'espaces cloisonnés ou interconnectés de tailles variées plus riche que le simple inventaire des rues et des bâtiments laisserait deviner. La « Petite ville » - la traduction littérale de Lillköping - offre en fait un vaste champ de possibilités.

La ville du polar est souvent un espace purement fictionnel qui emprunte aux clichés du dédale et du labyrinthe pour souligner sa littéralité, transformant l'enquête sur un crime en une quête du récit et ainsi de sens. ${ }^{21}$ Lillköping se prête aussi à cette lecture, mais ici la pratique de l'espace est davantage autotélique. Certes, les enquêtes de Kalle 
et le jeu de la Guerre des Roses sont des activités orientées vers une fin (la possession durable de Stormumriken signifierait la victoire ultime), mais l'être véritable des jeunes protagonistes se manifeste dans ces activités elles-mêmes indépendamment de leur achèvement ainsi que dans les pratiques d'espace qu'elles créent. La ville avec sa structure de relations et de granularités variées et ses frontières multiples est appropriée et transformée par les enfants. Véritables tacticiens de l'espace dans le sens que Michel de Certeau a donné à cette notion, Kalle, Eva-Lotta et Anders jouent dans les jardins, préparent des opérations contre les Roses rouges dans leur quartier général au grenier, grimpent dans les arbres et les toits pour espionner ou surveiller, et s'aventurent dans la prairie ou le château à la recherche de suspense. À travers ces pratiques, ils modifient la ville; leur activité crée dans l'espace, pour utiliser les expressions de Certeau, « de l'ombre et de l'équivoque », «y insinue [une] multitude de références et citations" et le transforme "en singularités grossies et en îlots séparés $»^{22}$. En investissant ainsi les différents lieux de la ville de significations qui proviennent de leurs activités, de leurs jeux fondés sur l'imagination, ils construisent un lieu porteur de leur propre identité : Kalle, Eva-Lotta et Anders sont, d'une manière fondamentale, des habitants de cette ville de jeu et d'aventure; leurs valeurs, leur histoire, leurs projets, leur éthique de camaraderie, leur maitrise du corps et des nerfs, testés dans les aventures, leur être même se manifeste dans cet espace et s'épanouit dans l'utilisation créative des possibilités qu'il offre.

L'espace propre des enfants reste cependant entrelacé avec le monde des adultes ; il est une construction éphémère et mouvante en contact permanent avec son extérieur. Le simple fait d'être rattrapé par les obligations familiales - comme arroser les fraises ou tenir le magasin de son père - suffit pour ramener l'enfant à son rôle subordonné dans la société. Ces moments de contact donnent souvent lieu à une négociation de l'identité où l'enfant est examiné et évalué à l'aide de critères habituellement utilisés dans le monde des adultes, comme l'âge, l'intelligence, la maturité - et que l'enfant peut contester. Quand Eva-Lotta est interrogée par la police en qualité de témoin qui a vu un homme suspecté de meurtre, elle doit se défendre contre l'approche dépréciative du commissaire qui ne la connaît pas :

«Bonjour, petite Lisa-Lotta », dit le commissaire avec énergie.

«Eva-Lotta », dit Eva Lotta. « Bonjour!»

« Bien sûr, oui - Eva-Lotta ! Viens t'asseoir ici, petite Eva-Lotta, on va parler un peu.

Ça ne va pas prendre beaucoup de temps et ensuite tu pourras jouer de nouveau avec tes poupées"

Et il dit cela à Eva-Lotta, qui se sentait si vieille, presque quinze ans !

"J'ai fini de jouer avec les poupées il y a dix ans", dit Eva-Lotta en guise d'éclaircissement.

Constable Björk avait raison - celle-ci était manifestement une enfante ancrée dans

la réalité. Le commissaire comprit qu'il fallait changer de ton et traiter Eva-Lotta comme si elle était une adulte. ${ }^{23}$

Ce qui est intéressant dans cette citation et dans le passage qui suit n'est pas uniquement le fait que le commissaire doive ajuster son comportement après avoir sérieusement sous-estimé la jeune témoin, mais aussi la réaction d'Eva-Lotta ellemême. Elle se sent vieille - presque 15 ans !-, mais ce sentiment n'est pas uniquement positif. Bien qu'elle revendique le droit d'être respectée, elle ne veut pas devenir adulte tout d'un coup non plus. Elle veut rester de son côté de la frontière qui sépare le monde des enfants de celui des adultes. Elle a cette possibilité, et elle lui est précieuse, car c'est elle qui lui donne la force de continuer après les événements traumatisants qu'elle a 
vécus. Dix pages plus loin, le narrateur constate qu'« Il est vrai qu'elle se sentait vieille, comme si elle avait presque 15 ans, mais elle avait heureusement la capacité d'un jeune esprit d'oublier ce qui était désagréable presque d'un jour à l'autre $»{ }^{24}$

Le monde des enfants et celui des adultes sont imbriqués et les contacts entre les deux sont continus, engendrant d'autres négociations où l'un exerce une influence sur l'autre. Après deux jours d'absence qui ont suivi la collecte du témoignage par la police, Eva-Lotta recommence à jouer à la Guerre des Deux-Roses avec ses amis pour mieux oublier le crime qu'elle a vu. Elle ressort de la maison familiale et repart en aventure dans la ville et dans ses alentours, dans l'espace propre des enfants. Plus tard, elle, Kalle et Anders se retrouvent tout d'un coup tout seuls avec le meurtrier dans un manoir abandonné situé à la lisière de la ville. À ce moment, c'est dans leur identité fantasmée de chevalier de la Rose Blanche qu'ils trouvent le courage et les astuces pour se confronter à la situation. Le jeu offre ainsi aux trois amis les moyens psychologiques et pratiques qui leur permettent de survivre dans le monde des adultes, tout en restant des enfants. Les possibilités offertes par l'hybridation des genres que nous avons indiquée plus haut se trouvent ainsi exploitées dans un espace romanesque qui a plusieurs caractéristiques en même temps : il est terrain de jeu, d'aventure, d'histoire imaginée et de crimes réels - et seuls les enfants ont accès à tous ces volets. Tout le long de la série, le "être enfant" se manifeste de cette manière: par un savant positionnement par rapport à la société des adultes - ses normes, conceptions, règles, pratiques - et par une utilisation inventive de l'espace qui offrent ensemble la possibilité de puiser dans le fantasme, l'imagination, le jeu et la camaraderie pour affronter la réalité, même sous ses apparences les plus sombres.

\section{L'héritage de Kalle Blomkvist}

Victoire du jeu, de l'imagination et de l'innocence donc? Oui, peut-être. La perturbation que le crime apporte à Lillköping est l'occasion d'une épreuve où le monde des enfants - du jeu, de l'aventure - entre en contact avec le monde des adultes. Les trois amis réussissent l'épreuve que cette expérience de frontière engendre grâce à leur amitié et leur courage, mais aussi grâce au jeu et à leur maîtrise de l'espace de la ville qu'ils s'approprient d'une manière créative ; l'épreuve devient ainsi une occasion pour affirmer leur identité dans toute sa complexité et aider la petite ville à retrouver son ordre social. La série sur Kalle Blomkvist propose ainsi une lecture de la société où l'intégration, l'harmonie et la paix sont des valeurs dominantes, mais qui souligne en même temps l'autonomie des enfants. Elle repose sur la conception de Lillköping et de la Suède comme des espaces à part, bien que la frontière qui permet de les démarquer soit ambiguë.

Comme nous l'avons indiqué au début de l'article, Fifi Brindacier et d'autres romans de Lindgren continuent à susciter plus d'intérêt que la série sur Kalle Blomkvist, surtout dans la recherche. Les aventures du jeune détective continuent cependant à être lues, et le nom du protagoniste apparaît souvent dans des mémoires d'auteurs reconnus et dans des recensions qui jaugent la littérature contemporaine pour les enfants. ${ }^{25}$ Son héritage est toujours présent. L'œuvre dispose d'une modernité poétique et aborde des thèmes qui sont toujours d'actualité. L'hybridation des genres, le particularisme suédois ou nordique accompagné d'une anxiété concernant les frontières, la métatextualité, les pratiques de l'espace et les négociations identitaires sont des 
éléments essentiels du polar contemporain et se trouvent également fréquemment dans d'autres genres. La littérature contemporaine les aborde dans un contexte différent marqué par l'émergence des sociétés plus multiculturelles (la Suède l'a toujours été), l'éclipse du nationalisme suivi de sa résurgence, et l'intensification des échanges culturels et commerciaux à l'échelle globale. Le Lillköping de Kalle n'existe plus. Elle a été une métonymie pour réfléchir sur les relations sociales et intergénérationnelles dans le folkhemmet et sur la place de la Suède dans le monde de la guerre froide. Dans ce sens, elle a préparé la voie aux défis des décennies suivantes que le polar cherche à soulever.

\section{NOTES}

1. Auteurs respectivement de Barnets århundrade (Le Siècle de l'enfant, 1900) et Ett hem (Un Foyer, 1899).

2. Voir par exemple les statistiques dans Johan Svedjedal, « Svensk skönlitteratur i världen », in Johan Svedjedal (éd.), Svensk litteratur som världslitteratur. En antologi, Uppsala, Avdelning för litteratursociologi vid Uppsala universitet, 2012, p. 48-52.

3. Index Translationum de l'UNESCO, http://www.unesco.org/xtrans/ ; Astrid Lindgren - Werk und Wirkung. Internationale und interkulturelle Aspekte, Svenja Blume, Bettina Kümmerling-Meibauer et Angela Nix (éds.), Frankfurt am Main, Peter Lang (collection Kinder- und Jugendultur, -literatur und -medien. Theorie-Geschichte-Didaktik, 60), 2009 ; Denis Ballu, Lettres nordiques. Une bibliographie, 1720-2013, vol. II, collection Acta Bibliothecae regiae stockholmiensis LXXXVIII.

4. Caroline Roeder, "Archivalisches zur Astrid Lindgren-Rezeption in der DDR", in Svenja Blume, Bettina Kümmerling-Meibauer et Angela Nix (éds.), op.cit. , p. 105-122 ; Mare Müürsepp, «Die Werke Astrid Lindgrens in der estnischen Kinderkultur. Von der Vermittlung zur Assimilation », ibid., p. 175-189.

5. Helena Forsås-Scott, Swedish Women's Writing, 1850-1995, London, Athlone Press (coll. Women in Context), 1997 ; Maria Nikolajeva, «Literature for Children and Young People », in Lars G. Warme (éd.), A History of Swedish Literature, Lincoln, Nebraska University Press, 1996, p. 500 ; Thierry Maricourt, Voyages dans les lettres suédoises, Nantes, L'Élan, 2007, p. 62 ; Boel Westin, La Littérature enfantine en Suède, trad. Lydie Rousseau et Denis Béhar, Paris, Institut suédois, 1998, p. 25 ; Beyond Pippi Longstocking. Intermedial and International Aspects of Astrid Lindgren's Works, Bettina Kümmerling-Meibauer et Astrid Surmatz (éds), New York, Routledge, 2011.

6. Läs mig! Sluka mig!, Kristin Hallberg (éd.), Stockholm, Natur och Kultur, 1998 ; Maria BergbomLarsson, « Astrid Lindgren - en kärleksförklarning », in Ingrid Holmquist et Ebba Witt-Brattström (éds), Kvinnornas Litteraturhistoria, vol. II, Malmö, Författarförlaget, 1983, p. 276-293 ; Lena Kåreland, Skönlitteratur för barn och unga. Historik, genrer, termer, analyser, Lund, Studentlitteratur, 2015, p. 86-87.

7. Voir Eva Söderberg, "L'héritage de Fifi Brindacier en Suède ", Cahiers du Genre, n 49, 2010, p. 77-96.

8. Si le lien entre les deux personnages masculins peut apparaître assez superficiel, une espèce de clin d'œil au lecteur, la parenté entre Fifi et Lisbeth Salander est plus profonde, voir Andrew Nestingen, «Unnecessary Officers. Realism, Melodrama and Scandinavian Crime Fiction in Transition », in Scandinavian Crime Fiction, Andrew Nestingen et Paula Arvas (éds.), Cardiff, 
University of Wales Press, 2011, p. 171-183. Eva Söderberg voit également une parenté entre les personnages, voir Söderberg, op. cit., p. 95.

9. L'expression « kulturell resonansbotten » vient de Johan Svedjedal, op. cit., p. 57.

10. Voir Lena Kåreland, Modernismen i barnkammaren. Barnlitteraturens 40-tal, Stockholm, Rabén \& Sjögren (Skrifter utgivna av Svenska Barnboksinstitutet, 66), 1999 ; Angela Nix, "Chaos, Spiel und Akrobatik. Astrid Lindgren im Kontext des schwedischen Modernismus ", in Svenja Blume, Bettina Kümmerling-Meibauer et Angela Nix (éds.), op. cit., p. 17-33.

11. Ulf Boëthius, «Wild, ill-mannered and disobedient. Pippi Longstocking and the debate on uncivilized youth and bad children ", in Svenja Blume, Bettina Kümmerling-Meibauer et Angela Nix (éds.), op. cit., p. 55-66.

12. Northrop Frye, Anatomy of Criticism, Princeton, Princeton University Press, 1957.

13. «Sådant hände helt enkelt inte. Inte här. I Amerika kanske - det hade man läst i tidningarna om - men inte här. » Astrid Lindgren, Kalle Blomkvist och Rasmus, Stockholm, Rabén \& Sjögren, 1984, p. 33. Toutes les traductions de sources originales par l'auteur de l'article.

14. « Hon tar in alltsammans med blicken och låter den sedan vandra vidare ut genom vindrutan. Det är ett svensk sommarlandskap de jagar fram igenom, inget tvivel om den saken. De mogna rågfälten med prästkragar och blåklint i är så svenska som helst. De vita björkstammarna också. Det är bara den här bilen och dess underliga passagerare som inte hör hemma där, de hör hemma i en amerikansk gangsterfilm. [...] Kidnappare - det verkar så fånigt i ett sånt här solig svenskt sammanhang! », ibid., p. 39.

15. Thèmes qu'on trouve par exemple dans Organes vitaux (2012) et United Victims (2010) d'Elsebeth Egholm, Mon premier meurtre (1993) de Leena Lehtolainen, la série Stockholm noir I-III (2006-2011) de Jens Lapidus, les séries télévisée Broen (2011-2018) et Bedrag (2016-2019), pour ne mentionner que quelques exemples.

16. Siegfried Krakauer, Le roman policier. Un traité philosophique, Paris, Payot et Rivages (collection Petite bibliothèque Payot), 2001 (orig. 1922-1925), p. 32.

17. Voir par exemple Maria Lang, Inte flera mord!, Stockholm, Norstedts, 1951.

18. Westin, op. cit., p. 26.

19. "Varför skulle en del människor ha sån tur att födas i Londons slumkvarter eller i Chicagos förbrytarkretsar, där mord och skottlossning hörde till ordning för dagen ? ", Astrid Lindgren, Mästerdetektiven Blomkvist, Stockholm, Rabén \& Sjögren, 1985, p. 3.

20. " "Jo", medgav Anders, "sånt där som händer oss, det händer bara i böcker." "Det kanske är bara en bok det här", sa Kalle. "Vad menar du, är du inte klok", sa Anders. "Vi kanske inte finns", sa Kalle drömmande. "Vi kanske bara är ett par killar i en bok, som nån har hittat på." "Ja, du möjligen?", sa Anders förargad. "Skulle inte förvåna mej, om du vore ett tryckfel, när allt kommer omkring. Men inte jag, det ska jag bara be att få tala om !" "Det vet du inte", vidhöll Kalle. "Du kanske bara finns i en bok som jag hittat på." "Kyss mej”, sa Anders. "I så fall finns du i en bok som jag har hittat? på, och jag börjar ångra att jag nånsin hittade på dej." "Förresten är jag hungrig”, sa Kalle. » Astrid Lindgren, Kalle Blomkvist och Rasmus, p. 47-48.

21. Marion François, «Le Stéréotype dans le roman policier », Cahiers de Narratologie [en ligne], $\mathrm{n}^{\circ} 17,2009, \mathrm{p} .4-5$. Consulté le 30 septembre 2016.

22. Michel de Certeau, L'invention du quotidien. 1. arts de faire, Paris, Gallimard (collection Folio essais), 1990, p. 152-153, voir aussi p. 59-63 sur l'utilisation tactique de l'espace.

23. «"Gomorron, lilla Lisa-Lotta", sa kriminalkommissarien hurtigt. "Eva-Lotta", sa Eva-Lotta. "Gomorron !" “Javisst, ja - Eva-Lotta ! Kom och sätt dej här, lilla Eva-Lotta, så ska vi pratas vid ett slag. Det kommer inte att ta lång stund, och sen får du gå leka med dina dockor igen.” Och detta sa han till Eva-Lotta, som kände sig så gammal, nästan som femton år ! "Jag slutade leka med dockor för tio år sen”, sa Eva-Lotta upplysningsvis. Konstapel Björk hade rätt - det var tydligen ett sakligt barn det här. Kommissarien förstod att han måste lägga om ton och handla Eva-Lotta 
som en vuxen. » Astrid Lindgren, Mästerdetektiven Blomkvist lever farligt, Stockholm, Rabén \& Sjögren, 1984, p. 75.

24. «Visserligen kände hon sig ju gammal, nästan femton år, men hon hade lyckligtvis ett ungt sinnes förmåga att glömma det som var obehagligt nästan från den ena dagen till den andra. », ibid., p. 85.

25. Voir par exemple Leif G. W. Persson, Gustavs grabb - berättelsen om min klassresa, Stockholm, Bonniers, 2011 ; Bertil Falk, «Björn Hellberg: Deckare och fotboll lika oförutsägbart », DAST Magazine. Tidskrift för underhållningslitteratur, janvier 12, 2008, http://www.dast.nu/artikel/bjornhellberg-deckare-och-fotboll-lika-oforutsagbart, consulté le 20 décembre 2019; et Dan Sjögren, «För mycket av Kalle Blomkvist», Norrtelje Tidning, $1^{\text {er }}$ décembre 2015, https:// www.norrteljetidning.se/artikel/for-mycket-av-kalle-blomkvist, consulté le 20 décembre 2019.

\section{RÉSUMÉS}

Dans l'œuvre d'Astrid Lindgren, les aventures du maître détective Kalle Blomkvist publiées entre 1946 et 1953 semblent avoir moins bien résisté au temps que les récits classiques sur Fifi Brindacier ou Ronya la fille de brigand. La série propose cependant une modernité narrative et des thèmes qui présentent un intérêt pour l'étude de la littérature suédoise et nordique, et notamment le polar. Un lien explicite mène en fait des trois volumes Blomkvist à la série Millennium de Stieg Larsson qui reprend deux figures d'Astrid Lindgren, Kalle et Fifi, pour en proposer de nouvelles interprétations dans le couple asymétrique de Mikael Blomkvist et Lisbeth Salader. Au-delà de ces références, on peut observer d'autres types de connexions entre la série et le polar contemporain, notamment dans la conception de la Suède comme un espace à part moralement supérieur et protégé de l'influence extérieure ainsi que dans l'hybridation de genres, la métatextualité et l'importance accordé à l'espace urbain et à la négociation de l'identité.

Among Astrid Lindgrens works, the three books on the master detective Kalle Blomkvist published between 1946 and 1953 seem to attract less attention today than the classic works on Pippi Longstocking or Ronia the Robber's Daughter. The series contains however narrative modernity and themes that are interesting for the study of Swedish and Nordic literature and especially of the fashionable genre of Nordic noir. Not only is there an explicit connection between characters in the three books on Blomkvist and in Stieg Larsson's Millenium -series, but the series is related to Nordic noir also by its reflection on Sweden as a morally superior country, by its hybridisation of genres and metatexuality, and by the importance it offers to the urban space and the negotiation of identity.

\section{INDEX}

Mots-clés : littérature jeunesse, Suède, Lindgren (Astrid)

Keywords : children's literature, Sweden, Lindgren (Astrid) 


\section{AUTEUR}

\section{HARRI VEIVO}

Harri Veivo est professeur au département d'études nordiques et directeur de l'équipe d'accueil 4254 ERLIS à l'université de Caen Normandie. Ses recherches portent sur la littérature et la culture des pays nordiques du $19^{\mathrm{e}}$ siècle à nos jours. Il est l'auteur par exemple de « En jaguar in den europeiska djungeln. Tre dikter av Elmer Diktonius i internationella sammanhang i början an 1920-talet » (Historiska och litteraturhistoriska studier, 93, 2018), « Fracas, bruit et fanfares pour la victoire. Interprétations du jazz et de la modernité dans les pays nordiques » (Deshima, 13, 2019) et éditeur avec Petra James et Dorota Walczak-Delanois de Beat Literature in a Divided Europe (Leiden, Brill, 2019). 